\title{
SERPENT2-SUBCHANFLOW-TRANSURANUS PIN-BY-PIN DEPLETION CALCULATIONS FOR A PWR FUEL ASSEMBLY
}

\author{
Manuel García $^{1}$, Riku Tuominen ${ }^{2}$, Andre Gommlich ${ }^{3}$, Diego Ferraro ${ }^{1}$, Ville Valtavirta ${ }^{2}$, \\ Uwe Imke $^{1}$, Paul Van Uffelen ${ }^{4}$, Luigi Mercatali ${ }^{1}$, Victor Sanchez-Espinoza ${ }^{1}$ \\ Jaakko Leppänen ${ }^{2}$, Sören Kliem ${ }^{3}$ \\ ${ }^{1}$ Karlsruhe Institute of Technology (KIT) \\ Hermann-von-Helmholtz-Platz 1, 76344 Eggenstein-Leopoldshafen, Germany \\ ${ }^{2}$ VTT Technical Research Centre of Finland Ltd. \\ Tietotie 3, Espoo, FI-02044 VTT, Finland \\ ${ }^{3}$ Helmholtz-Zentrum Dresden-Rossendorf (HZDR) \\ Bautzner Landstraße 400, 01328 Dresden, Germany \\ ${ }^{4}$ Joint Research Centre (JRC), European Commission (EC) \\ Hermann-von-Helmholtz-Platz 1, 76344 Eggenstein-Leopoldshafen, Germany \\ manuel.garcia@kit.edu, Riku.Tuominen@vtt.fi, a.gommlich@hzdr.de
}

\begin{abstract}
This work presents the results for a coupled neutronic-thermalhydraulic-thermomechanic pin-level depletion calculation of a PWR fuel assembly using Serpent2-SUBCHANFLOWTRANSURANUS. This tool is based on a semi-implicit depletion scheme with pin-by-pin feedback, mesh-based field exchange and an object-oriented software design. The impact of including fuel-performance capabilities is analyzed, with focus on high-burnup effects. The treatment of the Doppler feedback to the neutronics is examined as well, in particular the use of radial fuel-temperature profiles or radially averaged values.
\end{abstract}

KEYWORDS: Serpent2, SUBCHANFLOW, TRANSURANUS, Multiphysics, PWR.

\section{INTRODUCTION}

As part of a global trend to develop high-fidelity applications for reactor physics simulations, the EU Horizon $2020 \mathrm{McSAFE}$ project [1] was set to develop multiphysics tools based on the Monte Carlo particle transport method, with the objective of tackling large-scale pin-by-pin depletion and transient problems.

In this framework, a coupling scheme for Serpent2 [2], a continuous-energy Monte Carlo code, SUBCHANFLOW (SCF) [3], a subchannel thermalhydraulics code, and TRANSURANUS (TU) 
[4], a fuel-performance code, has been developed with the aim at performing high-fidelity depletion calculations [5]. The motivation to add fuel-performance analysis capabilities to the standard neutronic-thermalhydraulic approach is the improvement of the modelling of relevant fuel phenomena, such as fission gas release and fuel-cladding gap behavior, and of fuel temperatures for Doppler feedback to the neutronic calculation. The implementation and main features of this threecode coupling are described in Section 2.

In this work, Serpent2-SCF-TU is applied to a pin-level depletion calculation of a typical PWR fuel assembly, presented in Section 3. The results with and without TU are shown and compared to assess the impact and potential gains of adding fuel-performance analysis to the traditional scheme. In addition, the effect of the Doppler feedback to Serpent 2 using averaged and radially dependent fuel temperatures is studied. These results, presented in Section 4, serve to assess two main issues identified in previous studies, namely the behavior of the fuel solution for high-burnup states and the potential improvement of the fuel temperature feedback to the neutronic calculation using an improved thermomechanic model and more detailed temperature distributions [5].

\section{SERPENT2-SUBCHANFLOW-TRANSURANUS}

The Serpent2-SCF-TU calculation scheme is based on the standard neutronic-thermalhydraulic iterative method, with the simplified fuel-rod solver in SCF replaced by the more accurate thermomechanic analysis methodology in TU. Figure 1a shows the coupled depletion and feedback methodology.

For the neutronic calculation the standard thermalhydraulic feedback is used, i. e. the coolant density $\rho_{\text {cool }}$ and temperature $\mathrm{T}_{\text {cool }}$ from SCF and the fuel temperature $\mathrm{T}_{\text {fuel }}$ from TU are used. For $\mathrm{T}_{\text {fuel }}$ either a volume average, an effective value of the form $\mathrm{T}_{\text {fuel }}^{\text {eff }}=\mathrm{w}_{\text {fuel }}^{\text {surface }}+(1-\mathrm{w}) \mathrm{T}_{\text {fuel }}^{\text {centine }}$, with some weight $\mathrm{w}$, or the actual radial profile $\mathrm{T}_{\text {fuel }}(\mathrm{r})$ can be used. SCF simulates only the coolant conditions and provides the clad-coolant heat transfer coefficient $h_{\text {clad-cool }}$ and the coolant temperature $\mathrm{T}_{\text {cool }}$ and pressure $\mathrm{p}$ to $\mathrm{TU}$, which performs the fuel calculation with these fields as boundary conditions. All the feedback is done at pin-level for each axial zone.

The depletion scheme is semi-implicit, in the sense that each burnup step is iterated and the fields at the end of the step are used as feedback and to evaluate the convergence. Each depletion iteration from step $t_{n}$ to $t_{n+1}$ consists of a steady-state SCF calculation at $t_{n+1}$, and TU and Serpent 2 burnup steps from $t_{n}$ to $t_{n+1}$. TU simulates each step as a transient with an internal time discretization, performing the thermomechanic calculation and solving a simplified set of Bateman equations with a low-order approximation for the neutronic parameters. Each Serpent 2 iteration consists of the solution of the Bateman equations using the Stochastic Implicit Euler (SIE) method, a type of relaxation, and a transport calculation at $t_{\mathrm{n}+1}$.

The object-oriented development strategy used for the three-code coupling is shown in Figure $1 \mathrm{~b}$. Each code is restructured as a calculation library and wrapped into a C++ class that defines the multiphysics interface. These classes are derived through inheritance from an abstract base class which represents a template of a code suitable for multiphysics simulations, with a well-defined interface. In addition, the format used to set and get variables to and from the codes is defined by the MEDCoupling library, which implements unstructured meshes and fields defined on them. Using the generic base class and the MEDCoupling meshes and fields, the three codes are handled 
in the exact same way and exchange feedback with the same format, which greatly facilitates the implementation of the coupling scheme, which is specified in a separate "supervisor" program.

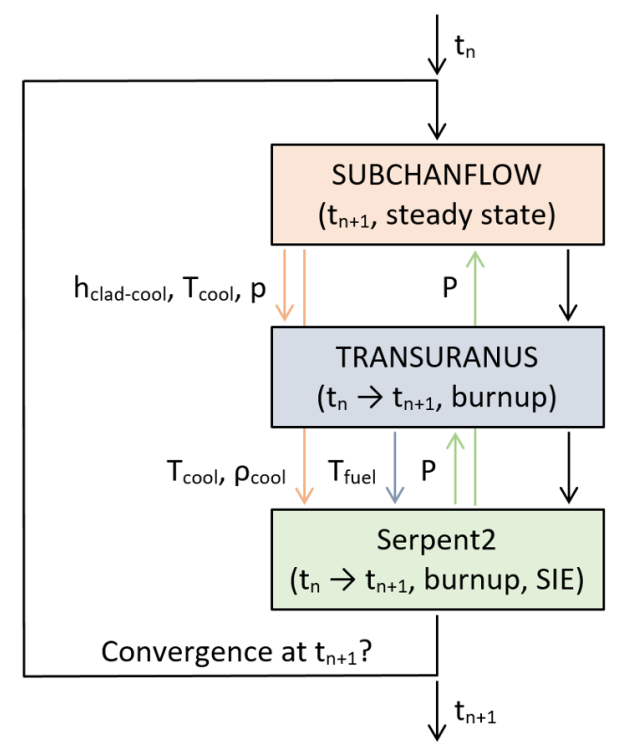

(a)

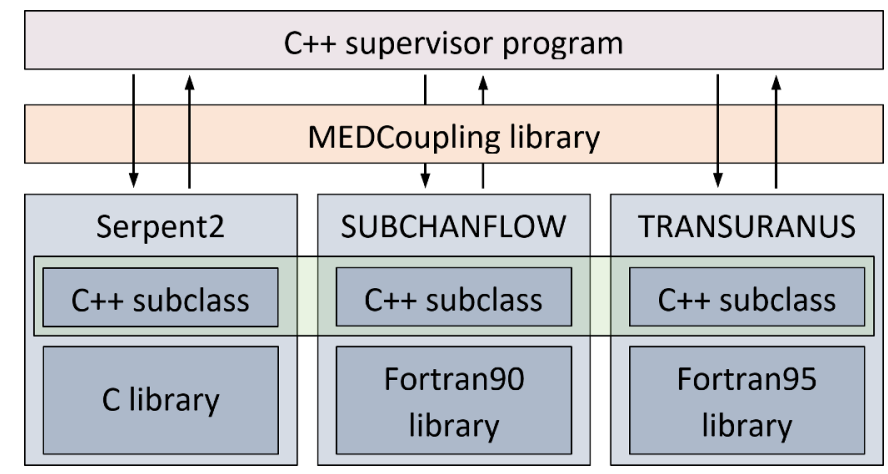

(b)

Figure 1: Depletion and feedback scheme (left), object-oriented software design (right).

\section{TEST PROBLEM AND MODELLING APPROACH}

The test case considered is problem 6 of the VERA Core Physics Benchmark Progression [6]. It consists of a Westinghouse $17 \times 17$ fuel assembly with 24 guide tubes and a single instrumentation tube, defined at Beginning of Cycle (BOC) and Hot Full Power (HFP) in the benchmark. A 1305day $(51.84 \mathrm{MWd} / \mathrm{kgU})$ burnup cycle at constant power and operating conditions is studied.

The Serpent 2 pin lattice is shown in Figure 2a, while the axial model, which consists of sections with and without grid spacers and of axial reflectors, can be seen in Figure $2 b$. Vacuum boundary conditions are used for the axial reflectors, with the problem reflected in the other directions. Each transport calculation is performed with 2,000 cycles of 100,000 neutrons, with the fission source calculated initially with 250 inactive cycles and corrected before each iteration with 50 cycles. For the depletion calculation 3 radial zones are used for all pins, and equilibrium Xe is considered.

Figure 2c shows the SCF subchannels, which correspond to a standard coolant-centered model. Spacer grids are modelled as local pressure losses. In the simulations without TU, the heat conduction equation in the fuel pins is solved using 10 radial nodes in the pellet and 2 in the cladding.

The TU model is comprised of all the fuel rods, leaving out the guide tubes. For each rod, 4 radial coarse zones for the fuel and 2 for the cladding are used, where thermomechanic properties are taken as uniform. Each of these zones is subdivided in 5 to 10 fine radial nodes. 


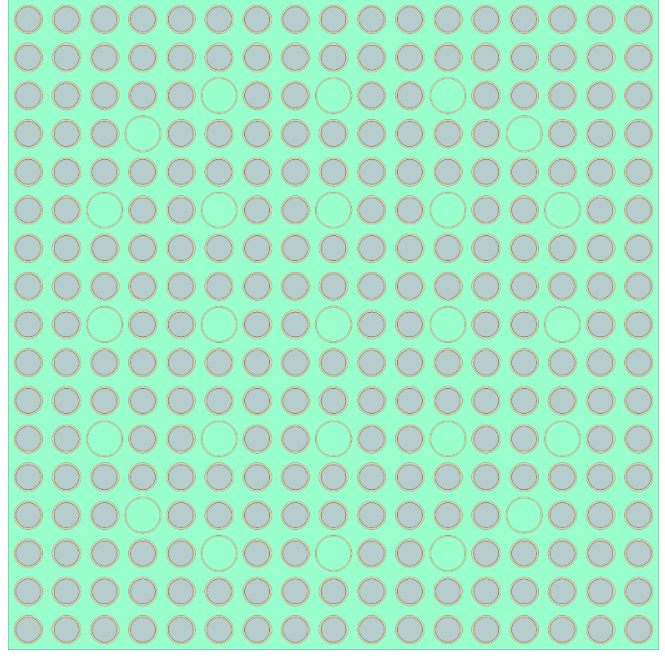

(a)

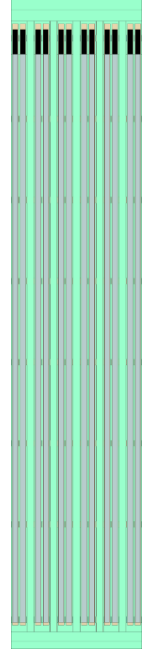

(b)

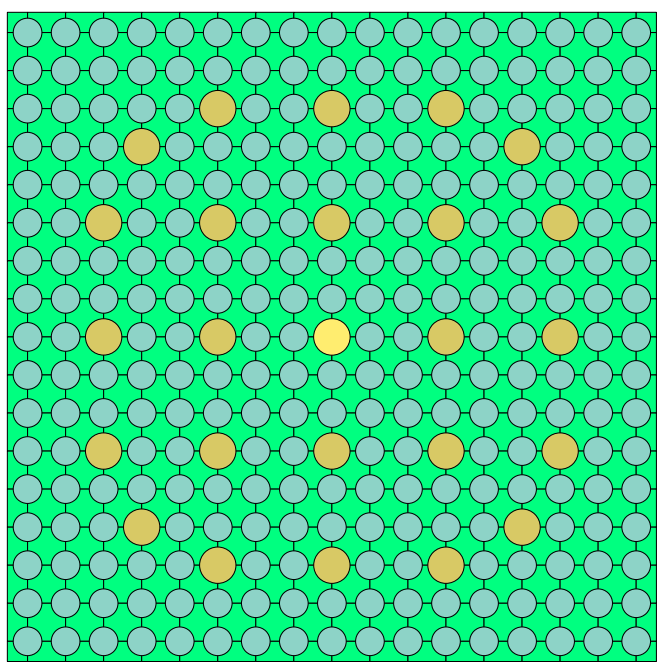

(c)

Figure 2: Serpent2 (left, center) and SCF (right) models.

\section{RESULTS AND DISCUSSION}

In this section, the results for Serpent2-SCF-TU (S2-SCF-TU) are shown and compared to Serpent2SCF (S2-SCF). In the three-code coupling the rods are simulated in TU and SCF only deals with the coolant solution, as detailed in Section 2. When TU is not used the rods are simulated in SCF using temperature-dependent thermal properties and a simple fuel-cladding gap model which includes thermal expansion and burnup-dependent fuel relocation by cracking and swelling. The Doppler feedback for the neutronic calculation is done using radial fuel temperature profiles. The differences of Serpent2-SCF with respect to Serpent2-SCF-TU are shown as maximum, mean or Root Mean Square (RMS) values over all rods and axial levels.

\subsection{Neutronic Solution and Effect of the Doppler Feedback Model}

The results for the multiplication factor for Serpent2-SCF with and without TU can be seen in Figure 3 , where the values obtained using volume-averaged fuel temperatures $\left(\overline{\mathrm{T}}_{\text {fuel }}\right)$ for Doppler feedback are included. In all cases the standard deviation is lower than $10 \mathrm{pcm}$. Overall the results for the four simulations are quite similar, and the largest differences can be observed at the end of the cycle, where the solutions without TU deviate up to $150 \mathrm{pcm}$ from the reference one. The effect of considering radially dependent fuel temperatures does not seem significant, at least not for the estimation of the multiplication factor.

The evolution of the peak factor $f_{P}$ and the differences in the power distribution are shown in Figure $4 \mathrm{a}$, while Figure $4 \mathrm{~b}$ shows the axial power profiles at 25.9 and $51.8 \mathrm{MWd} / \mathrm{kg}$ (End of Cycle, EOC). In both cases the results are in good agreement, and no clear trends resulting from the different fuel solvers can be observed. 


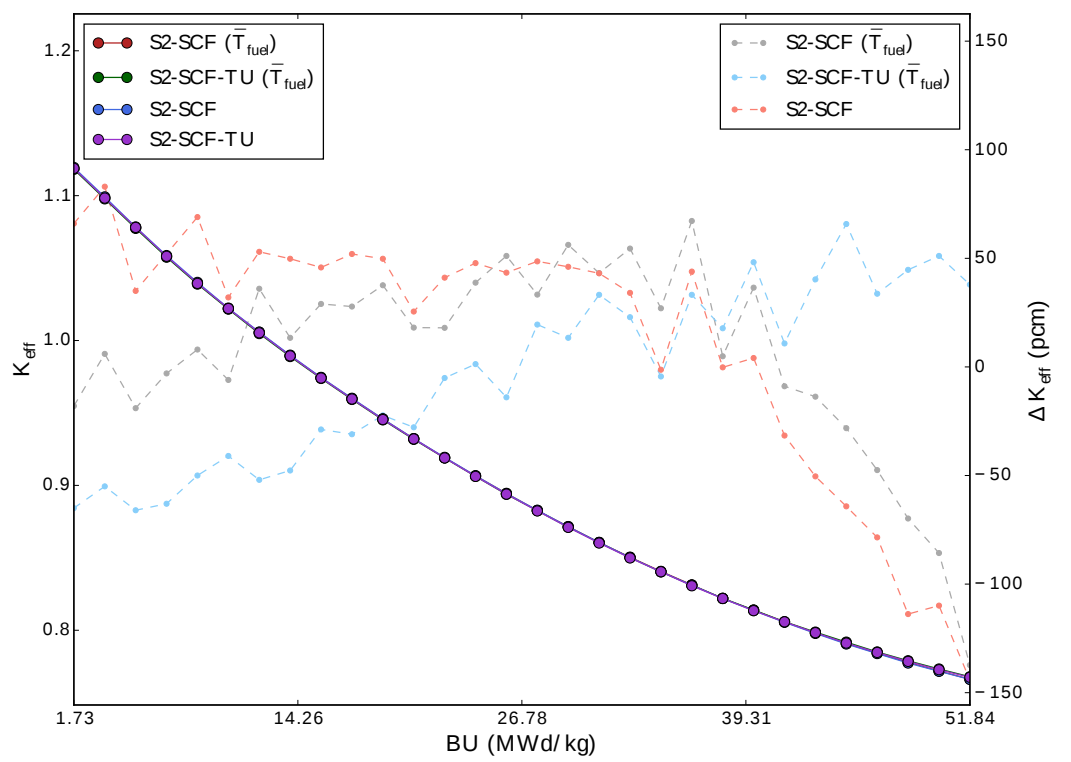

Figure 3: Multiplication factor.

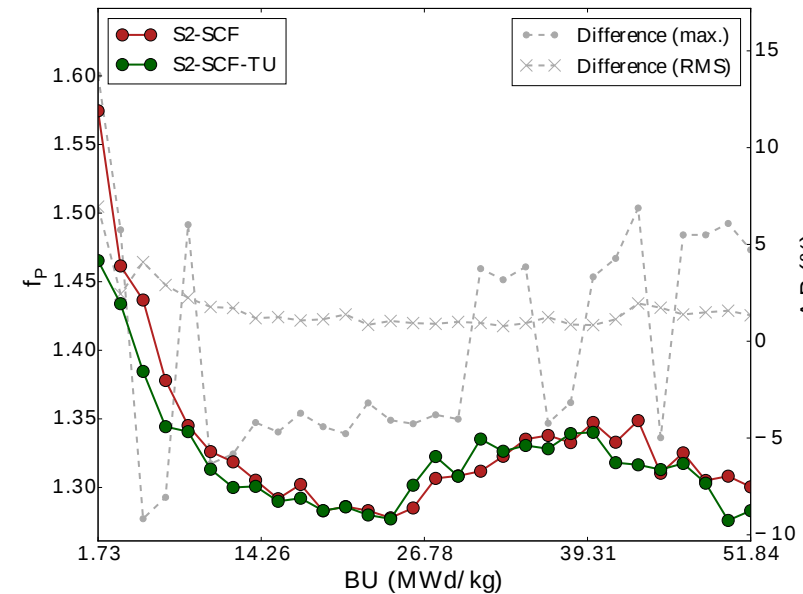

(a) Peak factor.

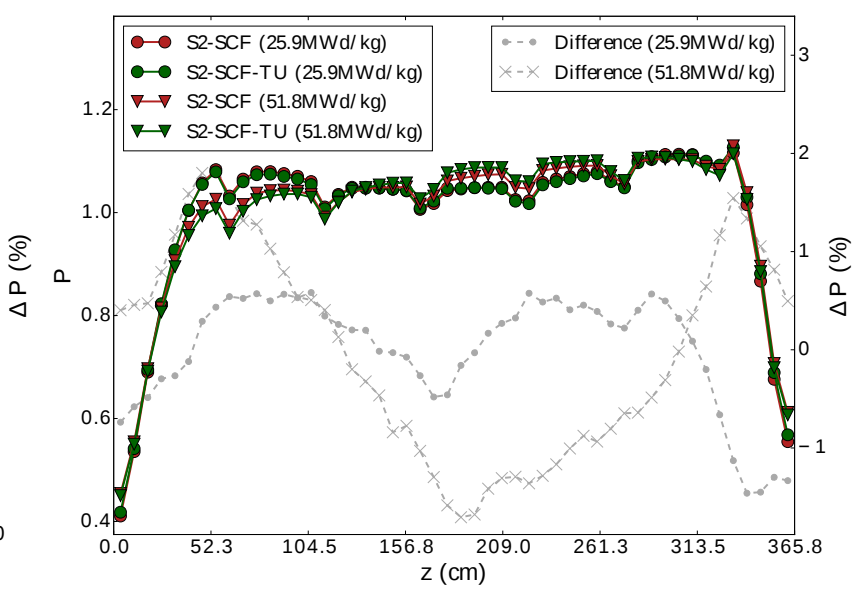

(b) Axial power profile.

Figure 4: Power.

\subsection{Thermomechanic Solution and Effect of the Fuel Model}

The global results for the fuel calculation with and without TU as a function of burnup are summarized in Figure 5. The cladding temperature (Figure 5a) is lower for SCF for the entire cycle, while the fuel outer temperature (Figure 5b) is higher. This larger temperature gradient in the fuelcladding gap for SCF can be attributed to the fact that SCF predicts significantly lower conductance (Figure 5e) and larger gap widths (Figure 5f). 
The largest differences are found in the rod centerline temperature (Figure 5c), where the average value calculated by SCF at EOC is about $350 \mathrm{~K}$ lower than the one predicted by TU. The average fuel temperature (Figure 5d) is underpredicted by about $175 \mathrm{~K}$ at EOC using SCF. This is a result of differences in the thermomechanic properties of the fuel, in particular the degradation of the conductivity due to burnup. In SCF the thermal conductivity depends only on the fuel temperature whereas in TU the dependency with burnup is included, resulting in a decreasing conductivity.

Figure 6 shows the radial temperature profiles at BOC and EOC for the hottest rod and axial position at these two states. The TU solution reflects the degradation of conductivity with burnup resulting in higher centerline temperatures, while in SCF these temperatures decrease due to the lower peak factor at higher burnup and to the fact that the conductivity does not depend on burnup.

\subsection{Pin-by-pin Results}

In addition to improving the modelling of the fuel behavior with respect to the traditional neutronicthermalhydraulic approach, the newly developed Serpent2-SCF-TU coupling allows the calculation of fuel-performance data using a fully coupled pin-by-pin approach. As an example, Figure 7 shows the fuel centerline temperature, the Xe release and the fuel-cladding gap conductance at EOC for this test case, all relevant safety parameters calculated with a novel high-fidelity approach.

\section{CONCLUSIONS}

A 51.84MWd/kgU burnup cycle for a PWR fuel assembly using Serpent2-SCF-TU was presented. This tool features a pin-by-pin semi-implicit depletion scheme, coupling Monte Carlo neutron transport, subchannel thermalhydraulic and fuel-performance analysis, and is based on replacing the simple fuel-rod solver in SCF with the full thermomechanic methodology in TU.

The treatment of the fuel temperature for Doppler feedback using averages or radial profiles was analyzed, and it was concluded that no significant changes are obtained in the depletion calculation increasing the detail in this aspect of the coupling. Relatively large differences in the fuel temperatures calculated by SCF and TU were observed, in particular for high-burnup states, where TU predicts centerline temperatures up to $350 \mathrm{~K}$ higher than SCF.

\section{ACKNOWLEDGMENTS}

This work was done within the McSAFE project which is receiving funding from the Euratom research and training programme 2014-2018 under grant agreement No 755097, and was performed on the computational resource ForHLR II funded by the Ministry of Science, Research and the Arts Baden-Württemberg and DFG ("Deutsche Forschungsgemeinschaft").

\section{REFERENCES}

[1] L. Mercatali, et al. "The EC McSAFE Project: High Performance Monte Carlo Methods for Safety Demonstration - Status and Perspectives." International Multi-Physics Validation Workshop, North Carolina State University, Raleigh, USA, June 14-15, 2018. 


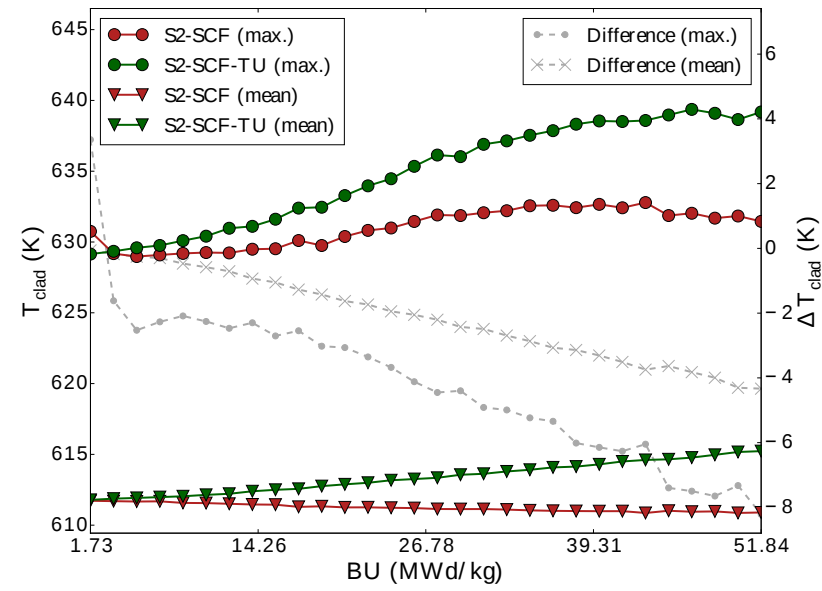

(a) Cladding temperature.

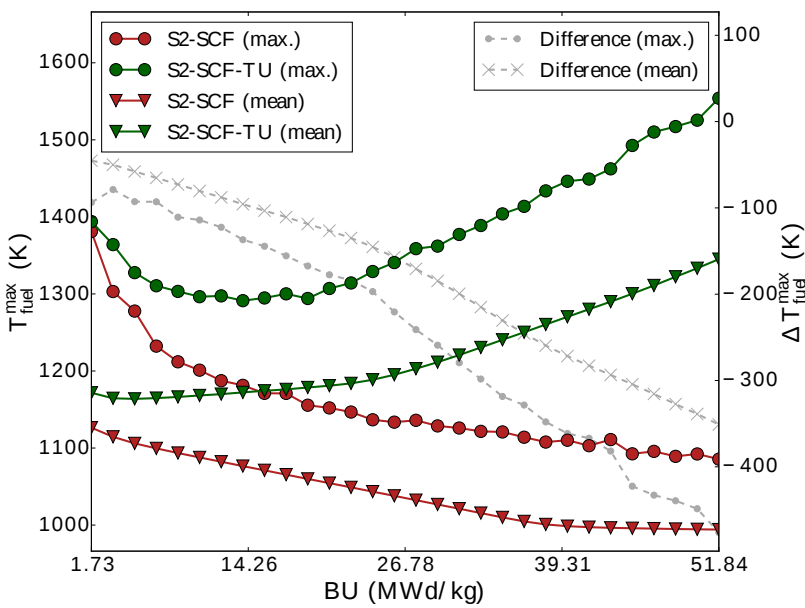

(c) Fuel centerline temperature.

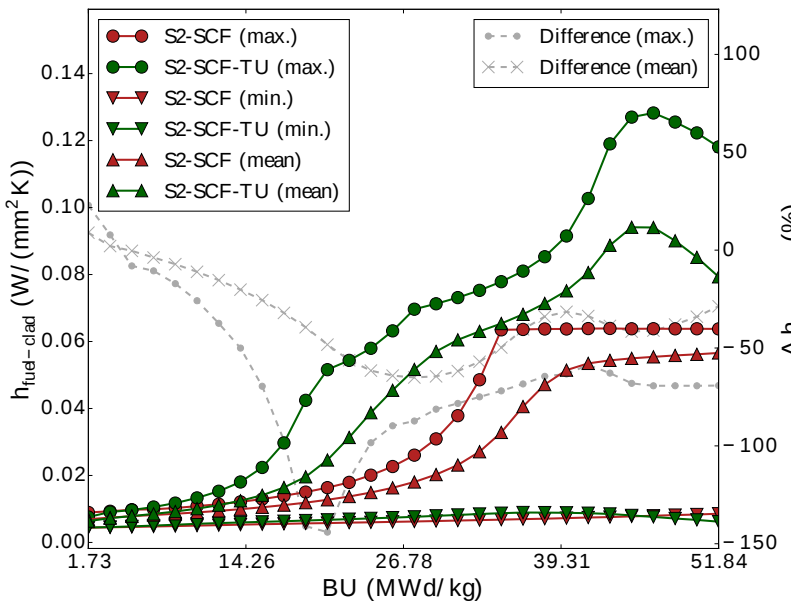

(e) Fuel-cladding gap conductance.

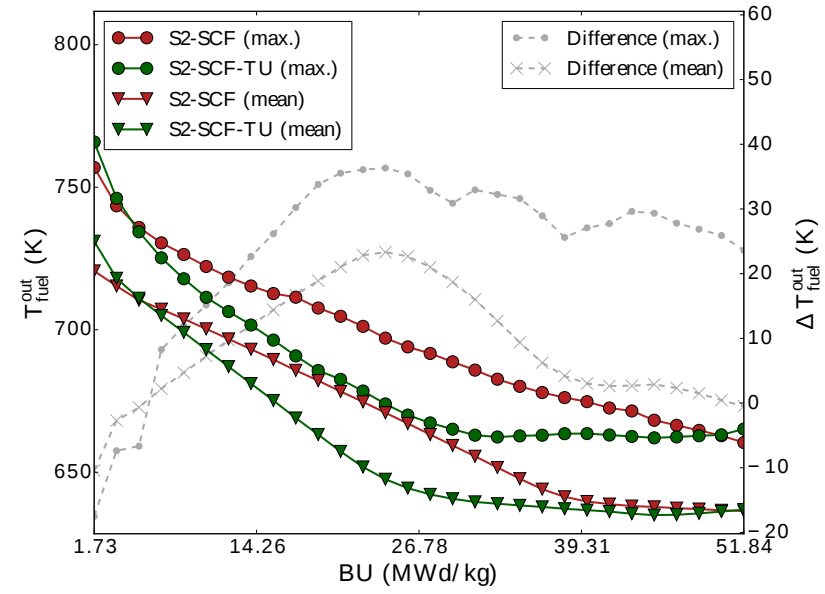

(b) Fuel outer temperature.

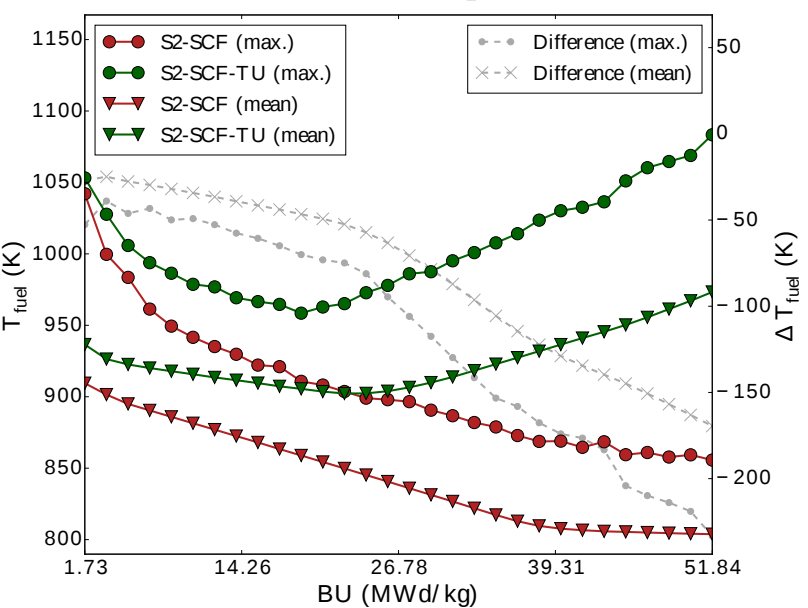

(d) Fuel average temperature.

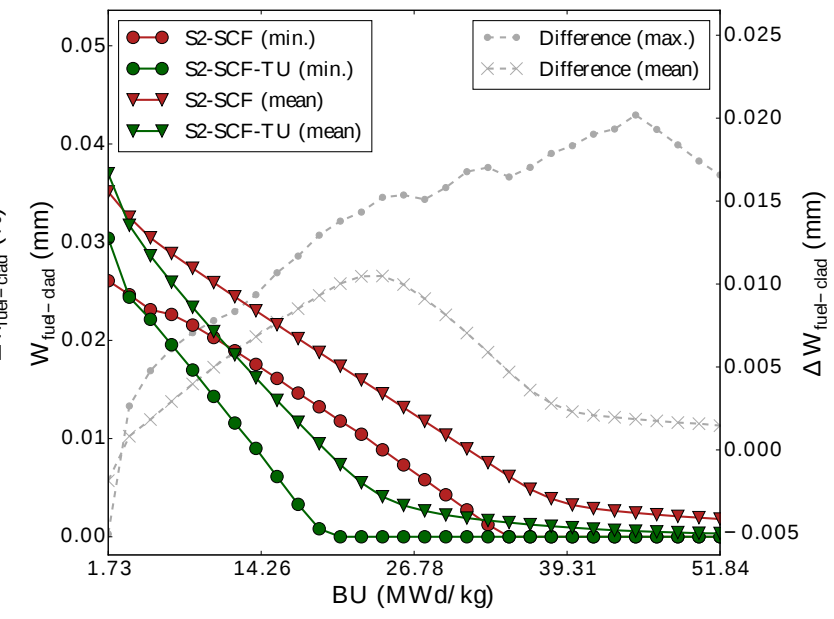

(f) Fuel-cladding gap size.

Figure 5: Fuel solution for Serpent2-SCF with and without TU. 


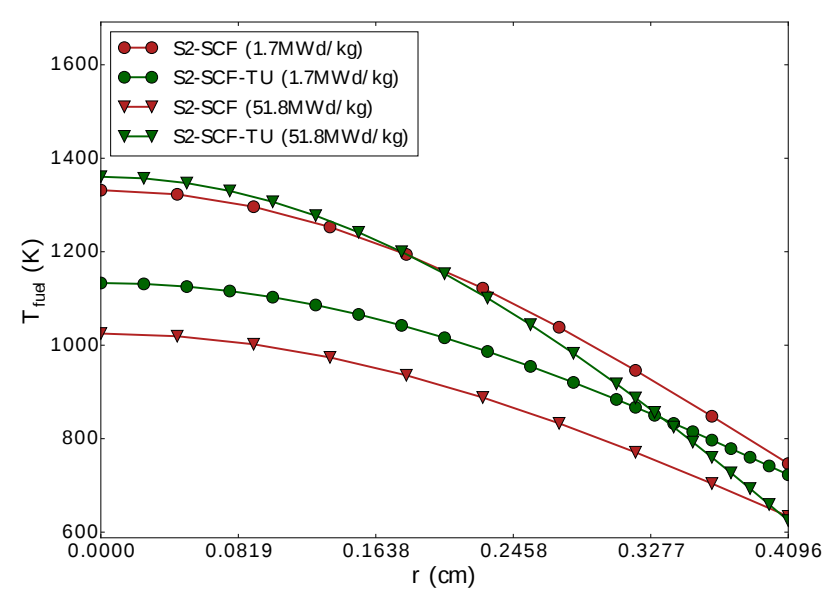

(a) Hottest rod at BOC.

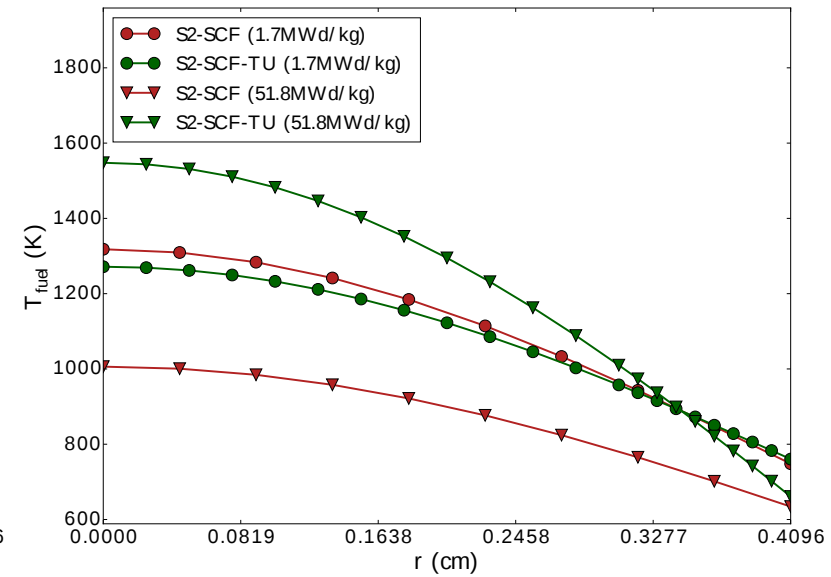

(b) Hottest rod at EOC.

Figure 6: Radial fuel temperature profiles.

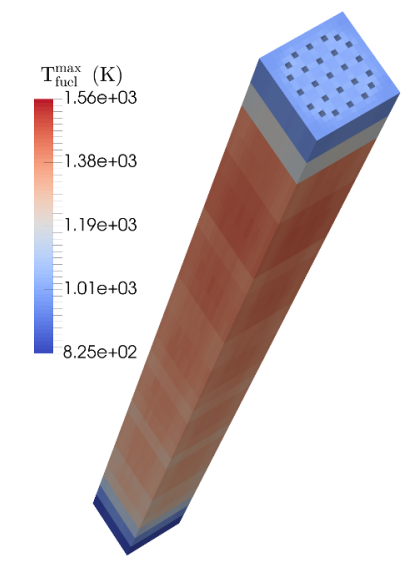

(a) Centerline temperature.

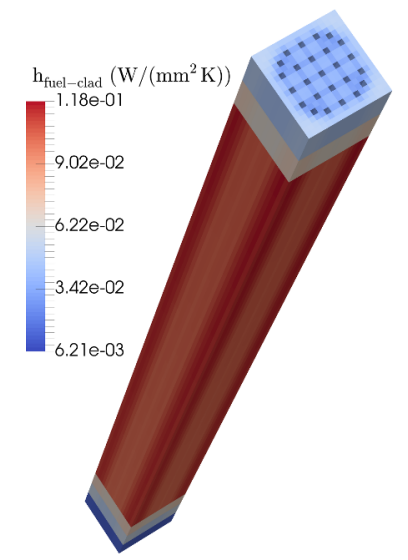

(b) Gap conductance.

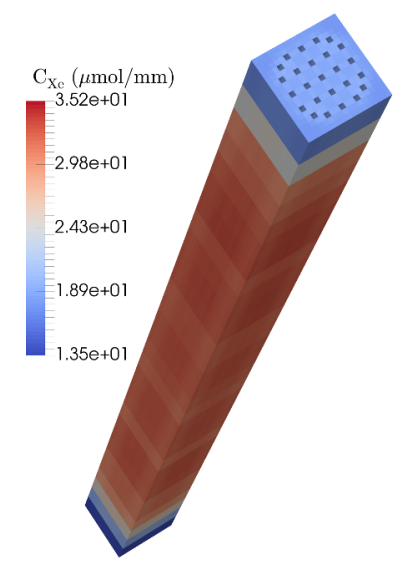

(c) Xe release.

Figure 7: Serpent2-SCF-TU pin-by-pin results at EOC.

[2] J. Leppänen, et al. "The Serpent Monte Carlo code: Status, development and applications in 2013." Annals of Nuclear Energy, volume 82, pp. 142-150 (2015).

[3] U. Imke, et al. "Validation of the Subchannel Code SUBCHANFLOW Using the NUPEC PWR Tests (PSBT)." Science and Technology of Nuclear Instalations, volume 2012 (2012).

[4] K. Lassmann. "TRANSURANUS: a fuel rod analysis code ready for use." Journal of Nuclear Materials, volume 188, pp. 295-302 (1992).

[5] M. García, et al. "A Serpent2-SUBCHANFLOW-TRANSURANUS coupling for pin-by-pin depletion calculations in Light Water Reactors." submitted to Annals of Nuclear Energy.

[6] A. Godfrey. "CASL - VERA Core Physics Benchmark Progression Problem Specifications." https://www.casl.gov/sites/default/files/docs/CASL-U-2012-0131-004.pdf. 